\title{
Acute otitis media in infants younger than three months not vaccinated against Streptococcus pneumoniae
}

\author{
Patricia Sommerfleck a,*, María Emilia González Macchi ${ }^{a}$, Silvana Pellegrini a , Patricia Bernáldez ${ }^{\text {a }}$, \\ Vanesa Reijtman ${ }^{\mathrm{b}}$, Claudia Hernández ${ }^{\mathrm{b}}$, Horacio Lopardo ${ }^{\mathrm{b}}$ \\ a Otolaryngology Department, Hospital de Pediatría SAMIC "Prof. Dr. Juan P. Garrahan", Combate de los Pozos 1881 CABA, Argentina \\ b Microbiology Department, Hospital de Pediatría SAMIC "Prof. Dr. Juan P. Garrahan", Combate de los Pozos 1881 CABA, Argentina
}

\section{A R T I C L E I N F O}

\section{Article history:}

Received 7 November 2012

Received in revised form 15 March 2013

Accepted 19 March 2013

Available online 17 April 2013

\section{Keywords:}

Acute otitis media

Infants

Younger than three months

\begin{abstract}
A B S T R A C T
The true incidence of acute otitis media (AOM) in neonates and infants younger than three months is unknown. In this population organisms causing AOM may disseminate leading to bacteremia, sepsis, and meningitis [1].

Objectives: To describe the clinical presentation, associated severe infections, bacteriologic etiology, and antibiotic resistance patterns of organisms isolated from the middle ear of infants younger than three months that were not vaccinated against Streptococcus pneumoniae.

Methods: Otomicroscopy was performed in all children to confirm the suspected diagnosis of AOM When purulent effusion retained in the middle ear was diagnosed, tympanocentesis and culture of middle-ear fluid was performed by conventional methods. Serotyping was done using the Quellung technique.

Results: From May 2, 2009 to February 28, 2010, 52 infants met the inclusion criteria. Thirty-six were male (69.2\%) and 16 were female (30.8\%). From these 52 patients, 76 samples were obtained for culture. Bilateral acute suppurative otitis media was diagnosed in $24(46.2 \%)$ infants. Of all infants, 18 (34.6\%) had been treated with antibiotics before tympanocentesis.

Eight patients (44.4\%) had negative middle-ear fluid cultures.

Sixty bacterial pathogens were isolated from the middle-ear fluid of 43 patients. Mixed infections were recorded in $14 / 52$ patients (26.9\%). Nine cultures were negative (17.3\%), of which eight were from patients that had previously been treated with antibiotics. S. pneumoniae was isolated from middle-ear aspirates of 26/52 (50\%) patients with acute suppurative otitis media. Twenty-two out of the 26 isolates were susceptible $(84.7 \%)$ and four were intermediately susceptible to penicillin (15.3\%). Streptococcus pyogenes was isolated in $3 / 52$ (5.8\%) and Haemophilus influenzae in $18 / 52$ patients (34.6\%). Five (27.8\%) of these were beta-lactamase producers. Blood cultures, cerebrospinal fluid, and urine cultures were negative. Parenteral antimicrobial treatment was indicated in 29/52 (56\%).

Conclusions: S. pneumoniae is the most frequent pathogen to cause AOM in this age group. Empirical treatment with amoxicillin or ceftriaxone should be considered depending on clinical suspicion of severe invasive infection.
\end{abstract}

(c) 2013 Elsevier Ireland Ltd. All rights reserved.

\section{Introduction}

Acute otitis media (AOM) is the most common bacterial infection in pediatric patients, however, the true incidence in neonates and infants less than three months (90 days) of age is unknown [2]. Technical difficulties in examining the external auditory canal (small and colapsable) and the tympanic membrane

\footnotetext{
* Corresponding author at: Cachimayo $1071^{\circ}$ “2”, 1424 CABA, Argentina Tel.: +54114432 2422 .

E-mail addresses: p.sommerfleck@gmail.com, patoysergio@hotmail.com (P. Sommerfleck).
}

may lead to failure to diagnose AOM, especially in young infants. Otomicroscopic examination is necessary in these patients to remove the cerumen. The anatomy of the mastoid and middle ear in infants (narrow aditus ad antrum and Eustachian dysfunction) and the inflammatory process of the mucoperiostium may cause blocking of the aditus ad antrum and trapping of exudates in the middle ear [3]. This stage of AOM is recognized as acute suppurative otitis media (ASOM). Subsequently, this stage of ear disease may be complicated with acute mastoiditis. In neonates and infants less than 12 weeks of age this infection may not be localized: it may disseminate leading to bacteremia, sepsis, and meningitis [1]. ASOM is an inflammation of the middle ear with purulent effusion retained in the middle ear with or without 
an intact tympanic membrane. The most common signs and symptoms are: bulging and erythema of the tympanic membrane, ear pain, fever, and/or otorrhea, which usually fail to improve after empirical antimicrobial treatment [4]. Tympanocentesis is considered the gold standard for the diagnosis of ASOM, to relieve pain, to identify the causative organisms by culture to determine the antimicrobial susceptibility pattern of isolated organisms to antibiotics [2], and to prevent serious complications in these very young patients.

The objective of this study was to describe the clinical presentation, associated severe infections, bacteriologic etiology, and antimicrobial resistance patterns of causative organisms through the prospective assessment of a confirmed first episode of ASOM in infants younger than three months at a pediatric tertiary care center over a period of nine months.

\section{Patients and methods}

Infants less than three months (90 days) of age with a confirmed first episode of ASOM were enrolled in a prospective study from May 2, 2009 to February 28, 2010. Febrile infants with irritability, poor feeding, restless sleep, vomiting, or diarrhea without an apparent source of infection were admitted to the pediatric emergency room or referred as outpatients by pediatricians to the otolaryngologist to confirm the suspected diagnosis of AOM using otomicroscopy. The different clinical stages of AOM - tubotympanitis and hyperemic, exudative, and suppurative AOM - were recognized by visualization of the tympanic membrane. Purulent effusion was suspected to be retained in the middle ear, with or without an intact tympanic membrane, when we observed bulging and erythema of the tympanic membrane showing opacity, thickening, and loss of anatomic references, as well as diminished or absent mobility, ear pain, fever and/or otorrhea.

Indications for tympanocentesis or myringotomy include the following [5-7]:

1- Otitis media in patients who are seriously ill or have toxic signs or symptoms.

2- Unsatisfactory response to antimicrobial therapy.

3- Onset of otitis media in a patient who is receiving antimicrobial agents.

4- Presence of suppurative complications.

5- Otitis media in the newborn, the very young infant, or the immunologically deficient patient, in each of whom an unusual organism should be suspected.

This procedure can usually be performed without general anesthesia. Adequate immobilization of the patient is essential. Tympanocentesis technique usually performed:

1. Cleaning the external auditory canal with $70 \%$ alcohol solution with boric acid to prevent bacterial contamination.

2. Tympanic membrane puncture may be performed in the prominent portion or anterior-inferior quadrant.

3. Aspiration of pus with a syringe with $1 \mathrm{~cm}^{3}$ of sterile saline solution.

4. Middle-ear samples are conserved in an oxygen-free atmosphere until their initial culture. Cultures are performed in sheep blood agar and in thioglycollate broth (both incubated at $35{ }^{\circ} \mathrm{C}$ in air) and in chocolate agar plates (incubated at $35{ }^{\circ} \mathrm{C}$ in $5 \%$ $\mathrm{CO}_{2}$ ). Anaerobic cultures are performed routinely in blood agar plates + vitamin $\mathrm{K}$ and anaerobic broth and incubated in an anaerobic jar. When purulent effusion retained in the middle ear was suspected, tympanocentesis and culture of middle-ear fluid (MEF) was performed with conventional techniques. In infants with bilateral otitis media both ears were sampled, and specimens from both middle-ear exudates were sent for culture [8]. Middle-ear fluid samples were collected in a transport medium and processed at the Microbiology Laboratory as previously described [4].

Pathogens were identified and antibiotic resistance patterns analyzed as commonly described [2]. Penicillin susceptibility tests were performed using the Etest (Biomérieux Argentina). Etest ${ }^{\circledR}$ is a predefined, stable gradient of 15 antibiotic concentrations on a plastic strip. Using innovative dry chemistry technology, Etest ${ }^{\circledR}$ is used to determine the on-scale minimum inhibitory concentration (MIC) of antibiotics, antifungal agents, and antimycobacterial agents. To define penicillin resistance the breakpoints of the Clinical and Laboratory Standards Institute (CLSI) for oral penicillin V were used. Performance Standards for Antimicrobial Susceptibility Testing are: Penicillin-susceptible S. pneumoniae: $\mathrm{MIC}=0.06 \mu \mathrm{g} / \mathrm{ml}$; Intermediately penicillin-susceptible S. pneumoniae: MIC between 0.125 and $1 \mu \mathrm{g} / \mathrm{ml}$; Resistant S. pneumoniae: $\mathrm{MIC}=2 \mu \mathrm{g} / \mathrm{ml}$ [9].

We defined a "mixed infection" as a coinfection with different pathogen strains grown in cultures of middle-ear fluid obtained from both ears in bilateral cases or in middle-ear fluid of the same ear in one infant.

Serotyping of $S$. pneumoniae isolates was performed with an initial screening using a latex reagent (Copenhagen Seruminstitut, Denmark) and then confirmed using the quellung reaction at the Instituto Nacional de Enfermedades Infecciosas (INEI) of the ANLIS "Dr Carlos G. Malbrán”, the reference center for pneumococcal serotyping in Argentina. Antisera were obtained from the Copenhagen Seruminstitut, Denmark. Only serotype 6C was identified using a polymerase chain reaction method (http:// www.cdc.gov/ncidod/biotech/strp/pcr.htm).

The identification of another source of infection in febrile infants required physical examination and/or positive cultures. Blood cultures were routinely obtained at the pediatric emergency room in all infants younger than three months admitted with a suspicion of severe bacterial infectious disease. Cerebrospinal fluid (CSF) and urine cultures were obtained when clinically indicated. The antibiotic regimens selected for treatment varied considerably according to culture results, the basis of disc sensitivities, and clinical signs and symptoms. The first choice for empirical antimicrobial treatment $[10,11]$ was amoxicillin or parenteral ceftriaxone depending on the symptoms of infection at onset in cases with negative cultures and confirmed ASOM. Infants were controlled at three follow-up visits two, seven, and 15 days after diagnosis and tympanocentesis. Clinical data were collected from the medical records of the patients.

Written informed consent to participate in the study was obtained from the parent or legal guardian of each child.

\subsection{Statistical analysis}

Data was analyzed with Excel, chi-square test to compare variables and Fisher's exact test. SPSS software (version 15.1 for Windows, SPSS, Inc., Chicago, IL) was used.

\section{Results}

During the study period 167 infants less than 90 days of age were evaluated to confirm or rule out AOM. Otomicroscopy was necessary to remove cerumen and visualize the tympanic membrane in $42 \%$ of the patients (70/167). Of all patients evaluated, 76\% (127/167) were diagnosed with different stages of AOM as the cause of fever and other symptoms. The first two stages of AOM (tubotympanitis and hyperemia) were diagnosed in $59 \%$ of infants and were treated with oral antibiotics. Purulent 
effusion was suspected to be retained in the middle ear in 52/127 (41\%) infants that met the inclusion criteria and tympanocentesis was performed. There were 36 male (69.2\%) and 16 female $(30.8 \%)$ infants. Distribution according to age was: less than one month (30 days) of age: 22 children (42.3\%), less than two months (60 days) of age: 18 children (34.6\%), and less than three months (90 days) of age: 12 children (23.1\%). Forty-seven of the 52 patients (90.4\%) were febrile when referred to the otolaryngologist to confirm presence or absence of AOM. Fifteen infants (28.8\%) were seen as outpatients. After the diagnosis of ASOM using tympanocentesis the infants were treated with oral antibiotics without the need for hospitalization. Following fever, the most common signs on admission were: irritability, poor feeding, and respiratory distress. More than one-third of the patients (34.6\%; 18/52) suffered from bronchiolitis as an associated illness. One 52-dayold infant was admitted because of fever and right facial paralysis (a recognized intratemporal complication of ASOM). Infants had been symptomatic for a mean of 2.3 days before admission (interquartile range: 1-4 days). Eighteen of the 52 children (34.6\%) had been treated with antibiotics previous to the diagnosis of ASOM. Fifteen of these 18 patients $(83.4 \%$ ) had received ceftriaxone, the first-choice treatment in febrile infants younger than three months. Of all children 55.8\% (29/52) had school-age siblings.

Data regarding breast-feeding were available for 39 infants of whom two (5.1\%) were bottle-fed and 37 (94.9\%) were breast-fed, with or without supplementation. None of the patients had been vaccinated against $S$. pneumoniae with the vaccine available at that time for children younger than 2 years old (Prevenar ${ }^{\circledR} 7$-valent).

\section{Microbiology}

Seventy-six samples of 52 patients were cultured. Bilateral ASOM was recorded in 24/52 (46.2\%) infants. In 5/24 (20.8\%) patients different bacteriological isolates grew in samples obtained from middle-ear fluid from both ears. Eighteen of 52 infants (34.6\%) had been treated with antibiotics before tympanocentesis and only 8/18 (44.4\%) had negative MEF cultures.

Sixty bacterial pathogens were isolated from the MEF of 43 patients. Mixed infections were recorded in 14/52 (26.9\%) of patients. Bilateral ASOM was diagnosed in 5/14 patients with more than one bacterial culture. In nine cases mixed cultures grew in samples of unilateral ASOM (Table 1). Nine cultures (17.3\%) were negative. Eight of them were obtained from patients who had received previous antimicrobial treatment. S. pneumoniae was isolated in middle-ear aspirates of a first episode of ASOM in 26/52
Table 2

Serotypes of Streptococcus pneumoniae.

\begin{tabular}{lcc}
\hline Serotype & No of isolates & Percentage \\
\hline 3 & 4 & 15.4 \\
4 & 1 & 3.8 \\
5 & 1 & 3.8 \\
8 & 1 & 3.8 \\
14 & 2 & 7.7 \\
$18 \mathrm{C}$ & 1 & 3.8 \\
$19 \mathrm{~A}$ & 4 & 15.4 \\
$19 \mathrm{~F}$ & 2 & 7.7 \\
$23 \mathrm{~A}$ & 1 & 3.8 \\
$23 \mathrm{~F}$ & 1 & 3.8 \\
$33 \mathrm{~F}$ & 1 & 3.8 \\
$7 \mathrm{~F}$ & 1 & 3.8 \\
$9 \mathrm{~V}$ & 3 & 11.5 \\
Other non-vaccinal serotypes & 1 & 3.8 \\
Unavailable isolates & 2 & 7.7 \\
Total & 26 & 100 \\
\hline
\end{tabular}

(50\%) patients. Almost 85\% (84.7\%; 22/26) of S. pneumoniae isolates were susceptible to penicillin, and 4/26 (15.3\%) were intermediately susceptible considering CLSI breakpoints for oral treatment [9]. Resistance to penicillin $V$ was not observed and all strains were found to be susceptible to penicillin $\mathrm{G}$, amoxicillin, and ceftriaxone by the Etest ${ }^{\circledR}$. The most prevalent serotypes were: 19A (15.4\%), 3 (15.4\%), 9V (11.5\%), 14 (7.7\%), and 19F (7.7\%) (Table 2). Intermediately susceptible $S$. pneumoniae belonged to serotypes $14(N=2)$, 19A $(N=1)$, and a non-vaccinal serotype $(N=1)$. In 18/52 (34.6\%) patients non-typable Haemophilus influenzae was isolated, five (27.8\%) of which were $\beta$-lactamase producers. S. pyogenes was isolated in 3/52 (5.8\%), Moraxella catarrhalis in 4/52 (7.7\%), S. aureus MS in 6/52 (11.5\%), S. aureus MR in 1/52 (1.9\%), Pseudomonas aeruginosa in $1 / 52(1.9 \%)$, and Proteus mirabilis in $1 / 52$ (1.9\%) (Table 3).

Eighteen of 52 patients suffered from bronchiolitis complicated by ASOM on admission. Bilateral ASOM was diagnosed in $50 \%$ of these 18 patients. Positive cultures of middle-ear fluid were obtained in $72.2 \%$ of cases (13/18). Mixed cultures were present in 3/18 (16.6\%). A coinfection of $S$. pneumoniae and $H$. influenzae was found to be the causative mechanism in $2 / 13$ (15.4\%) patients. Blood cultures were available in 32/52 (61.5\%) and urine cultures in 16/52 (30.7\%) infants. All blood and urine cultures were negative. Lumbar puncture was performed in 14/ $52(26.9 \%)$ patients and all CSF cultures were sterile. Moreover, CSF glucose and protein concentrations were within the normal parameters.

Table 1

Mixed pathogens isolated in 14 patients with unilateral or bilateral ASOM.

\begin{tabular}{|c|c|c|}
\hline Isolate right ear & Isolate left ear & Uni or bilateral culture \\
\hline S. pneumoniae Int/H. influenzae $\mathrm{BL}-$ & - & Unilateral \\
\hline S. pyogenes & S. pneumoniae S & Bilateral \\
\hline H. influenzae $\mathrm{BL}-/$ S. pneumoniae $\mathrm{S} /$ M. catarrhallis & H. influenzae $\mathrm{BL}-/$ S. pneumoniae $\mathrm{S}$ & Bilateral \\
\hline H. influenzae $\mathrm{BL}-/$ S. pneumoniae $\mathrm{S}$ & H. influenza $\mathrm{BL}-/ S$. pneumoniae $\mathrm{S} / \mathrm{S}$. aureus $\mathrm{SM}$ & Bilateral \\
\hline S. pneumoniae $\mathrm{S} / \mathrm{H}$. influenza $\mathrm{BL}+/ \mathrm{S}$. aureus $\mathrm{SM}$ & - & Unilateral \\
\hline P. mirabilis/S. pneumoniae $\mathrm{S}$ & - & Unilateral \\
\hline H. influenzae $\mathrm{BL}-/ S$. aureus $\mathrm{SM}$ & - & Unilateral \\
\hline H. influenzae $\mathrm{BL}+/ S$. aureus $\mathrm{SM}$ & - & Unilateral \\
\hline- & S. pneumoniae $\mathrm{S} / \mathrm{H}$. influenzae $\mathrm{BL}-$ & Unilateral \\
\hline H. influenzae $\mathrm{BL}-$ & H. influenzae $\mathrm{BL}-/$ S. pneumoniae $\mathrm{S}$ & Bilateral \\
\hline H. influenzae $\mathrm{BL}-/ S$. pneumoniae $\mathrm{S}$ & H. influenzae $\mathrm{BL}-$ & Bilateral \\
\hline H. influenzae $\mathrm{BL}-/ S$. pneumoniae $\mathrm{S}$ & - & Unilateral \\
\hline H. influenzae $\mathrm{BL}+/ \mathrm{S}$. pneumoniae $\mathrm{S}$ & - & Unilateral \\
\hline$S$ pneumoniae S/M. catarrhallis & - & Unilateral \\
\hline
\end{tabular}

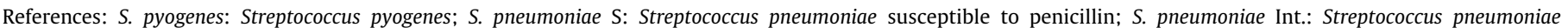

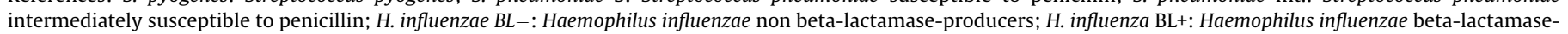
producers; M. catarrhalis: Moraxella catarrhalis; S aureus SM: Staphilococcus aureus susceptible to methicillin; P. mirabillis: Proteus mirabillis. 
Table 3

Bacterial isolates of 52 patients. In 14/52 (26.9\%) children mixed, polymicrobial cultures were obtained.

\begin{tabular}{lcc}
\hline Organism & No of isolates & Percentage \\
\hline Streptococcus pyogenes & 3 & 4.3 \\
Streptococcus pneumoniae & 26 & 37.7 \\
Haemophilus influenza & 18 & 26.1 \\
Moraxella catarrhalis & 4 & 5.8 \\
Staphylococcus aureus & 7 & 10.1 \\
Pseudomonas aeruginosa & 1 & 1.4 \\
Proteus mirabilis & 1 & 1.4 \\
Negative cultures & 9 & 13.0 \\
Total & 69 & 100 \\
\hline
\end{tabular}

\section{Antibiotic treatment}

The antibiotic regimens selected for treatment varied considerably according to culture results and clinical signs and symptoms. Initial empirical antimicrobial treatment was amoxicillin or parenteral ceftriaxone depending on the symptoms of infection at onset. When necessary (for example: in cases of $\beta$ lactamase-producing bacteria cultures) the first choice of treatment was changed and selected according to antibiotic resistance patterns of the isolated pathogens.

Twenty-one infants (40.4\%) were treated with oral amoxicillin; $1 / 52(1.9 \%)$ received oral amoxicillin/clavulanate, and 1/52 (1.9\%) was treated with oral ciprofloxacin.

Parenteral antimicrobial treatment was indicated in $29 / 52$ (56\%): 15 (28.8\%) were treated with ceftriaxone alone, 10 (19.2\%) with ceftriaxone and additional oral amoxicillin therapy after discharge, and $2 / 52(3.8 \%)$ received a combination of ceftriaxone and oral amoxicillin/clavulanate. Only 2/52 (3.8\%) required intravenous therapy with ceftazidime in one case and vancomycin in the other, because sepsis was suspected.

\subsection{Outcome}

The ASOM episode resolved in all patients and 45/47 became afebrile after tympanocentesis. Facial paralysis resolved two days after tympanocentesis in one case. Fifty-five percent of inpatients (22/40) was discharged within less than two days of hospitalization. Median hospital stay of children with respiratory distress and ASOM was six days (interquartile range: 2-8.25).

\section{Discussion}

ASOM might be underdiagnosed in febrile infants younger than three months because at this age otologic examination is technically difficult. Otolaryngologists and otomicroscopies are not always available in health care centers in developing countries. Other authors have observed the same in previous reports and recommend evaluation by an otolaryngologist with otomicroscopy [12]. In our study $42 \%$ of infants needed specialized evaluation to rule out an otologic source of infection because the ear canal was occluded by cerumen. Most cases of ASOM in infants aged younger than three months are caused by similar pathogens as those causing ASOM in older children. S. pneumoniae was found to be the most common pathogen in this group of infants, as in other studies [13-15].

Antibiotic resistance may already be present at an early age and should be considered when selecting the empirical treatment of ASOM in this population. In our study, $27.8 \%$ of $H$. influenzae isolates were $\beta$-lactamase producers. Less common bacteria (P. aeruginosa, $S$. aureus, and $P$. mirabilis) were isolated as clinically significant pathogens in $8 / 52$ cases of ASOM (15.4\%). Group A $\beta$-hemolytic streptococci (S. pyogenes) were isolated in $3 / 52(5.8 \%)$ cases in our study and should be considered as a possible pathogen in this group of infants. Other authors [16] have described S. pyogenes as the causative pathogen of ASOM in only $1.9 \%$ of children under 12 months of age. S. pyogenes is an important causative agent of invasive infections and local aggressiveness. Initial empirical antimicrobial treatment with amoxicillin or ceftriaxone should be recommended $[10,11,13-15,17]$. The choice of oral or parenteral antibiotics depends on the clinical presentation and suspected severe associated bacterial infections. In 5/24 (20.8\%) patients pathogens obtained from both ears were different. According to this finding, and in disagreement with other authors [2], we suggest that in bilateral ASOM, tympanocentesis should be done in both ears for culture. ASOM is a well-known complication in infants with bronchiolitis and we [17] recommend antimicrobial treatment in these cases. In our study, middle-ear cultures of infants suffering from bronchiolitis were positive in $72.2 \%$ of the cases.

The presence of ASOM does not predict a higher risk of severe bacterial infections in afebrile and febrile infants younger than three months of age if they receive appropriate treatment. None of our patients developed bacteremia or meningitis.

Febrile infants with an early diagnosis of ASOM were managed as outpatients in $44.2 \%$ of the cases. This modality saves costs of hospitalization and avoids complications associated with parenteral antimicrobial treatment.

\section{Conclusion}

Newborns and infants under three months of age may need specialized evaluation by otolaryngologist with otomicroscopy to rule out ASOM. S. pneumoniae is the most frequent pathogen of AOM in this age group. Empirical treatment with amoxicillin or ceftriaxone should be considered depending on clinical suspicion of severe invasive infection. Early diagnosis of ASOM in febrile infants allows early discharge and outpatient management in $44 \%$ of cases, saving hospitalization costs.

\section{Conflict of interest}

Nothing to declare.

\section{Acknowledgements}

The research leading to these results has received funding from the European Community's Seventh Framework Programme under Grant Agreement no. HEALTH-F3-2009-223111. This research was approved by the Ethical Committee and by the Dirección Asociada de Docencia e Investigación of the Hospital de Pediatría "Prof Dr Juan P Garrahan".

\section{References}

[1] D. Turner, E. Leibovitz, A. Aran, L. Piglansky, S. Raiz, A. Leiberman, et al., Acute otitis media in infants younger than two months of age: microbiology, clinical presentation and the therapeutic approach, Pediatr. Infect. Dis. J. 21 (2002) 669-674.

[2] W. Sakran, H. Makary, R. Colodner, D. Ashkenazi, Y. Rakover, R. Halevy, et al., Acute otitis media in infants less than three months of age: clinical presentation, etiology and concomitant diseases, Int. J. Pediatr. Otorhinolaryngol. 70 (2006) 613-617.

[3] V. Tarantino, D. Agostino, R. Taborelli, G. Melagrana, A. Pocu, A.M. Stura, Acute mastoiditis: a 10 year retrospective study, Int. J. Pediatr. Otorhinolaryngol. 66 (2002) 143-148.

[4] P.C. Bernáldez, G. Morales, L. Quantin, C. Hernández, J.L. Pinheiro, Otitis media aguda con retención de exudado purulento. Timpanocentesis diagnóstica y terapéutica. Estudio comparativo de la bacteriología con y sin tratamiento antibiótico previo en 189 niños y 288 oídos, Med. Infantil. 12 (2005) 97-102.

[5] B. Charles, S. Sylvan, Second edition, Pediatric Otolaryngology, vol. 1, W.B. Saunders Company, Philadelphia, USA, 1990, P390-392, 418-421.

[6] M.M., Paparella, D.A., Shumrick, Otorrinolaringología. Volumen 2. Segunda Edición. Editorial Médica Panamericana S.A. Junín 831,1 Piso. Buenos Aires, Argentina, 1982, 1424-1430. 
[7] A. Hoberman, J.L. Paradise, E.R. Wald, Tympanocentesis technique revisited, Pediatr. Infect. Dis. J. 16 (1997) S25-S26.

[8] P.A.Sommerfleck, P.C. Bernáldez, C.M. Hernández, R.V. Reijtman, H.A. Lopardo, Otitis media aguda: prevalencia de otopatógenos en pacientes de un hospital público, Acta Otorrinolaringol. Esp. 64 (2013) 12-16.

[9] Clinical and Laboratory Standards Institute. Performance standards for antimicrobial susceptibility testing, Eighteen Informational Supplement M 100-S 18, vol. 28, $\mathrm{N}^{\circ} 1,2008$.

[10] F. del Castillo Martín, F. Baquero Artigao, T. de la Calle Cabrera, M.V. Lopez Robles, J. Ruiz Canela, S. Alfayate Miguelez, et al., Documento de concenso sobre etiología, diagnóstico y tratamiento de la otitis media aguda, Ann Pediatr. (Barc.) 77 (2012), 345. e1-e8.

[11] Subcommittee on Management of Acute Otitis Media, Diagnosis, management of acute otitis media, Pediatrics 113 (2004) 1451, http://pediatrics.aappublications.org/content/113/5/1451.full.html.
[12] Y. Berkun, R. Nir-Paz, A. Ben Ami, A. Klar, E. Deutsch, H. Hurvitz, Acute otitis media in the first two months of life: characteristics and diagnostic difficulties, Arch Dis. Child. 93 (2008) 690-694. , http://dx.doi.org/10.1136/adc.2007.127522 (Originally published online 12 March).

[13] T.R. Tetzlaff, C. Ashworth, J.D. Nelson, Otitis Media in Children Less than 12 weeks of age, Pediatrics 59 (1977) 827-832.

[14] R.D. Bland, Otitis Media in the first six weeks of life: Diagnosis, Bacteriology, and management, Pediatrics 49 (1972) 187-197.

[15] D.M. Burton, A.B. Seid, D.B. Kearns, Neonatal otitis media. An update, Arch Otolaryngol Head Neck Surg 119 (1993) 672-675.

[16] N. Segal, N. Givon-Lavi, E. Leibovitz, P. Yagupsky, A. Leiberman, R. Dagan, Acute otitis media caused by Streptococcus pyogenes in children, CID 41 (2005) 35-41.

[17] M. Alapont, A. Gaiano, P. Bernáldez, G. Morales, L. Casimir, C. Hernández, et al., Etiología y características clínicas de la otitis media aguda en el ninio con bronquiolitis, Medicina Infantil IX (2002) 223-226. 